\title{
Assessment of Refractive Outcomes of Femtosecond-assisted Laser in Situ Keratomileusis (LASIK) for Hyperopia
}

\author{
Sadia Humayun ${ }^{1}$, Mazhar Ishaq ${ }^{2}$, Aisha Fawad ${ }^{3}$, Syed Fawad Mashhadi ${ }^{4}$, Quratulain Humayun ${ }^{5}$ and Sabahat \\ Arzoo $^{1}$ \\ ${ }^{1}$ Department of Refractive Surgery, Armed Forces Institute of Ophthalmology, Rawalpindi, Pakistan \\ ${ }^{2}$ Department of Eye, Mazhar Ishaq Centre of Ophthalmology, Rawalpindi, Pakistan \\ ${ }^{3}$ Department of Eye, Combined Military Hospital, Rawalpindi, Pakistan \\ ${ }^{4}$ Department of Community Medicine, Army Medical College, Rawalpindi, Pakistan \\ ${ }^{5}$ Department of Child and Family Asthma Studies Center, Women and Children's Hospital of Buffalo, NewYork, USA
}

\begin{abstract}
Objective: To determine the effect of refractive treatment in terms of safety, efficacy, stability, accuracy, and predictability with Wavefront Optimized Laser-assisted in situ keratomileusis (LASIK) on hyperopia.

Study Design: Descriptive, analytical study.

Place and Duration of Study: Armed Forces Institute of Ophthalmology, National University of Medical Sciences, Rawalpindi, Pakistan from August 2013 to June 2018.

Methodology: Participants were selected after detailed history, careful ocular examination and meticulous relevant assessment on refractive scans. Preoperative measurements were recorded. Refractive strategy was designed, considering individual requirements for each patient. Postoperative readings were taken at 1 day, 1 week, 1, 3, 6 and 12 months.

Results: A total of 54 patients participated with mean age of $28.07 \pm 8.13$ years. Out of the 54 patients, $33(61 \%)$ were males and 21 (39\%) were females. Uncorrected distance visual acuity (UDVA) and Spherical equivalent (SEQ), which is algebraic sum of sphere and half of cylinder, were recorded pre- and postoperatively. These parameters were changed significantly at 12 months $(p<0.001)$. SEQ reduced from $2.146 \pm 2.027$ to $0.079 \pm 0.269$ Diopter (D) $(p<0.001)$. UDVA improved from $0.672 \pm 0.270$ $(20 / 93)$ to $0.153 \pm 0.182(20 / 28)(p<0.001)$. A total of 48 (out of 54) patients had uncorrected vision better than 20/40; and 49 patients showed either same or gain of Snellen lines when pre- and postoperative corrected distance visual acuity (CDVA) was compared. Two patients showed loss of two or more lines. All the patients had postoperative SEQ within $\pm 1.0 \mathrm{D}$ range.

Conclusion: LASIK, performed to correct hyperopia, demonstrated satisfactory outcomes in terms of safety, efficacy, stability, accuracy, and predictability. LASIK is an effective treatment option for moderate $(<6$ D) hyperopia.
\end{abstract}

Key Words: LASIK, Refractive surgery, Hyperopia, Spherical equivalent, Uncorrected distance visual acuity.

How to cite this article: Humayun S, Ishaq M, Fawad A, Mashhadi SF, Humayun Q, Arzoo S. Assessment of Refractive Outcomes of Femtosecond-assisted Laser in Situ Keratomileusis (LASIK) for Hyperopia. J Coll Physicians Surg Pak 2021; 31(04):434-439.

\section{INTRODUCTION}

Hyperopia is a common refractive error, although it is less frequent than myopia. In middle ages, when the accommodation starts to wear out, hyperopes become more susceptible to spectacle dependence both for far as well as near. Laser-assisted in situ keratomileusis (LASIK) offers a reasonable spectacle free solution to these individuals. ${ }^{1,2}$

Correspondence to: Dr. Sadia Humayun, Department of Refractive Surgery, Armed Forces Institute of Ophthalmology, Rawalpindi, Pakistan

E-mail: sadia.humayun@yahoo.com

Received: June 06, 2020; Revised: February 14, 2021;

Accepted: March 08, 2021

DOI: https://doi.org/10.29271/jcpsp.2021.04.434
Hyperopia poses unique issues that merit altogether different approach to its refractive treatment in both young and middleaged. Higher levels of hyperopia can be associated with strabismus and amblyopia. Refractive surgery in such individuals can end up in troublesome situations like diplopia, resulting in a highly dissatisfied patient. ${ }^{3}$ Hyperopes are mostly under corrected due to variable degrees of accommodative excess they use for far distance, rendering difficulties in finding their appropriate refractive error. Therefore, it becomes important to cater for the true refractive error, which can be measured under cycloplegia.

LASIK does not prove to be an instant miracle for a hyperopic patient. Vision can take as long as one to three weeks to clear, till the accommodation relaxes. There are higher incidences of regression and need for retreatments reported with hyperopic corrections, possibly because of the change in the biome- 
chanics of cornea and a more prolate shape postoperatively. ${ }^{4,5}$ Some even advocate prophylactic corneal collagen crosslinking (CXL) with hyperopic treatments. ${ }^{6,7}$ Hyperopes exhibit larger angle kappa than myopes and emmetropes, which need special attention during LASIK. Angle kappa is the intersection between the line of sight and the pupillary axis. In hyperopes, pupil can be off centred to $1.5 \mathrm{~mm}$ from the visual axis. In such circumstances, ablation zone centration becomes critical which can be dealt with by pupil tracking systems in the newer generation machines and by shifting the flap nasally. ${ }^{8,9}$ Ablation algorithms like Wavefront Optimized profiles offer increased residual stromal tissue with minimal induction of spherical aberrations postoperatively. ${ }^{10,11}$

The purpose of this study was to find out the effects of Wavefront Optimized LASIKin hyperopia.

\section{METHODOLOGY}

This descriptive, analytical study was carried out at Armed Forces Institute of Ophthalmology from August 2013 to June 2018. After approval from the Institutional Review Board and Ethical Committee, patients undergoing the LASIK procedure were screened for eligibility criteria. Consent was taken from patients, who fulfilled the criteria; and one or both eyes were included through purposive sampling. Patients having hyperopia of spherical equivalent $6 \mathrm{D}$ or less, estimated postoperative steep $\mathrm{K}$ value $50 \mathrm{D}$ or less and minimum residual stromal bed thickness 280 microns were included in the study. Patients using contact lenses were advised to discontinue their use for a minimum of two weeks prior to initial assessment. Patients having age less than 18 years, unstable refraction, abnormal corneal topography, active corneal disease, lenticular opacities, other ocular pathologies, pregnancy and lactation, use of drugs like amiodarone and isotretinoin, systemic diseases like uncontrolled diabetes, autoimmune disorders, collagen vascular disorders or immune deficiency disorders were excluded from the study.

Preoperativeassessmentcomprised of a detailed history, examination including uncorrected distance visual acuity (UDVA), corrected distance visual acuity (CDVA), previous spectacle correction, cycloplegic refraction, slit lamp examination of both anterior and posterior segments, tonometry, dry eye assessment with tear film breakup time, investigations like corneal topography and pupillometry using Allegro Topolyzer vario, corneal tomography with Wavelight Oculyzer II and aberrometry with Wavelight Analyzer II, (by WaveLight $\mathrm{GmbH}$, Erlangen, Germany). Special emphasis was kept on cycloplegic refraction. ${ }^{12}$ Maximum tolerated plus was planned to be corrected. Patients were given refractive and contact lens trial for two weeks, where necessary to determine their appropriate target correction. Patients, who were found fit for the procedure, were given detailed counselling regarding the procedure by the refractive surgeon and counsellors. Patients were provided with reading material and shown videos about the procedure. Candidatesfor surgery were explained risk ofregression, use of presbyopic glasses, and possible need for retreat- ments. Written informed consent was taken from every patient before the procedure as per standard protocol.

All LASIK procedures were performed by either of two experienced surgeons. FS 200, 200 kHz Femtosecond Laser, (WaveLight $\mathrm{GmbH}$, Erlangen Germany) was used for flap creation and EX 500, $1050 \mathrm{~Hz}$ Excimer laser, (WaveLight GmbH, Erlangen Germany) was used for refractive ablation. Temperature and humidity standards were maintained as per the manufacturer recommendations. The attempted refractive result in all the procedures was aimed at emmetropia. Flap size was planned at 9.1 to $9.2 \mathrm{~mm}$, thickness varying from 100 to 120 microns with a side cut angle of 70 degrees. Standard optical zone was planned at $6.5 \mathrm{~mm}$ with ablation zone of $9.0 \mathrm{~mm}$ in most cases. Wavefront Optimized ablation profile was selected. This laser platform is equipped with high speed tracker system that recognises both pupil and limbus with the reaction time of three milliseconds. Nasal shift of pupil centre was checked by the system peroperatively on each patient and flaps were made more nasally using the displays provided on the machine display as shown in Figure $1 .{ }^{13}$ The hinge was kept supro-temporal in most cases to avoid interference with angle kappa and to minimise any interference with nasally shifted ablations. ${ }^{14}$ Pupil tracking system of the EX 500 system helped to overcome the challenge of nystagmus in one of the subjects.

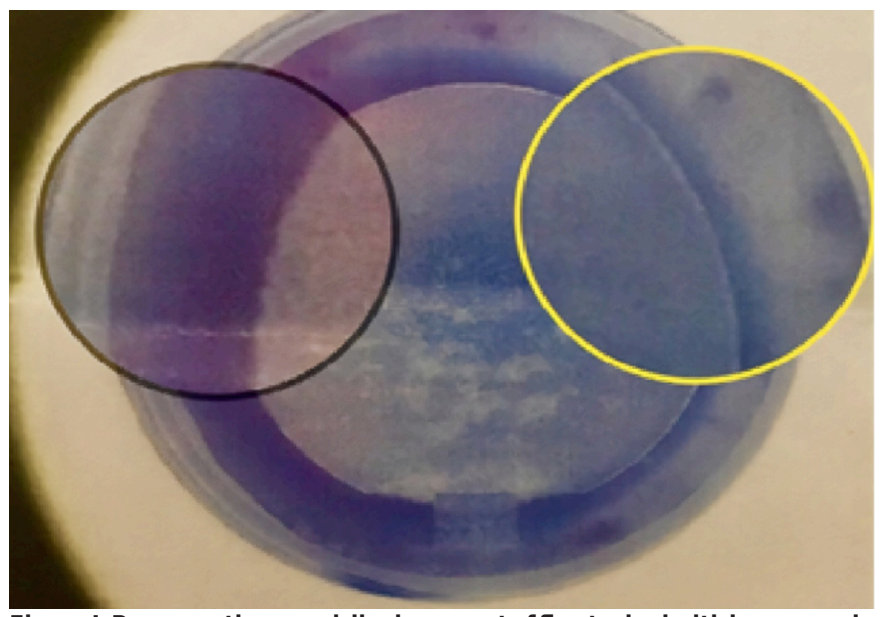

Figure l: Peroperative nasal displacement of flap to deal with larger angle kappa in hyperopes.

Standard surgical procedure, included patient positioning, instillation of topical anesthesia drops $(0.5 \%$ Proparacaine Hydrochloride eye drops), application of lid speculum, disinfection of ocular surface with povidone-iodine and thorough irrigation of conjunctival sac with balanced salt solution, placement of suction ring and application of Femto laser for making the flap. Excimer laser ablation was performed after lifting the flap followed by quickirrigation and repositioning of flap.

Postoperatively patients were examined before discharge for flap position and micro striae and prescribed tobramycin and dexamethasone combination drops 6-hourly for two weeks and Systane eye drops every two hours initially. Patients were given detailed briefing for post-operative care and were give handouts for the purpose. Patient follow-up was done at day 1, 1 
week then 1, 3, 6 and 12 months. On every visit, they underwent UCVA, tonometry and slit lampexamination. At six months, they had a tomography recorded.

The sample size was derived from the formula:

$$
n=\left(\frac{Z_{1 \frac{\alpha}{2} \times S D}}{d}\right)^{2}
$$

Where $Z_{1-\alpha / 2}$ represents the standard normal coefficient at $95 \%$ confidence interval (1.96), SD represents the standard deviation, and d represents the absolute error or precision level (0.5 D / 0.1 LogMAR). Using mean values for each anterior segment parameters that were previously reported in a study, various sample sizes were calculated and largest sample size was selected. The calculated total sample size was 36; and after adjusting for possibility of attrition, the final sample size was 54 . Overall, 54 study subjects were recruited into the study.

Statistical analysis was performed using IBM SPSS (version 25.0). Normality of data was assessed using Kolmogorov-Smirnov test. Qualitative data were expressed as frequency and percentage while quantitative as mean \pm SD. Paired sample two-test was used to compare the preoperative and postoperative data. Significance was set at $p$-value $<0.05$. The standard graphs for reporting outcomes of refractive surgery as suggested by the Journal of Cataract and Refractive Surgery, Cornea and Journal of Refractive Surgery were used. ${ }^{15,16}$ Simple linear regression analysis was carried out for attempted vs. achieved SEQ and target-induced astigmatism vs. surgically-induced astigmatism.

\section{RESULTS}

The mean age of the participants was $28.07 \pm 8.13$ years. Out of a total of 54 participants, $33(61 \%)$ were males and 21 (39\%) were females. UDVA, spherical equivalent SEQ and CDVA were recorded pre- and postoperatively as shown in Table I. Statistically significant difference was observed between pre- and postoperative UDVAand SEQ $(p<0.05)$. However, there was no statistically significant difference between pre- and postoperative CDVA. Comparison of postoperative values at 6 months and 12 months was done to observe changes during postoperative period. It was found that CDVA and UDVA showed a statistically significant change; however, SEQ had not changed significantly during this duration.

Efficacy profile was made by percentage of eyes that had achieved equal to or better UDVA compared with preoperative CDVA at 12 months (Figure 2A). Around $89 \%$ (48 out of 54) patients had UDVA equal to or better than $20 / 40$. Up to $76 \%$ (41 out of 54) participants had UDVA equal to or better than 20/30. Efficacy index (ratio of mean postoperative UDVA to mean preoperative CDVA) was calculated to be $0.96 \pm 0.15$.

Postoperative UDVA vs. preoperative CDVA profile was assessed by observing difference (loss or gain of lines) between UDVA and CDVA (Snellens lines) at 12 months as shown in Figure 2B.
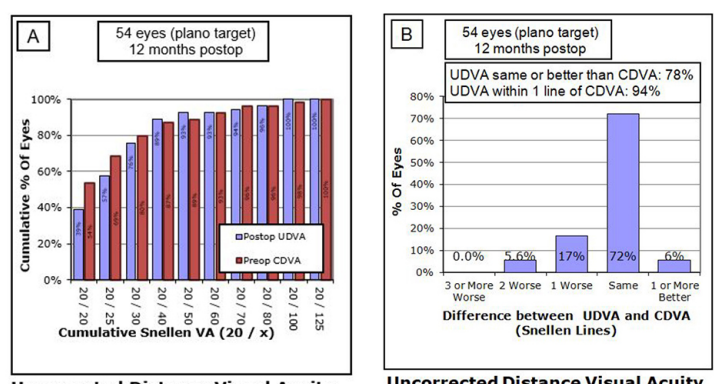

Uncorrected Distance Visual Acuity

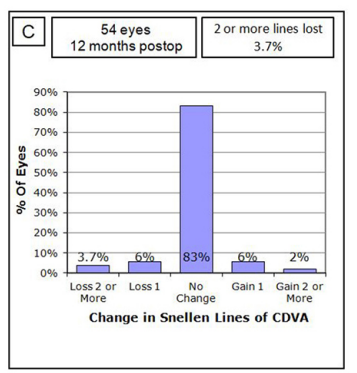

Uncorrected Distance Visual Acuity
vs. Corrected Distance Visual Acuity vs. Corrected Distance Visual Acuity

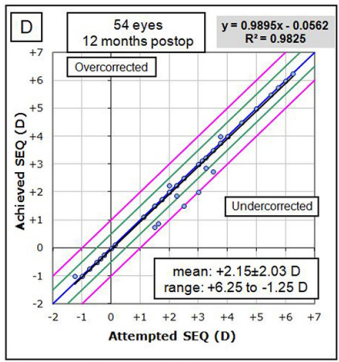

Change
Acuity

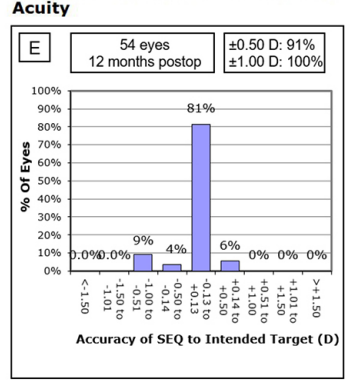

Spherical Equivalent Refraction
Accuracy

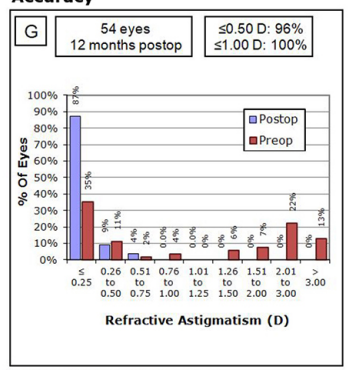

Spherical Equivalent Refraction

Spherical Equivalent Refrac
Attempted vs Achieved

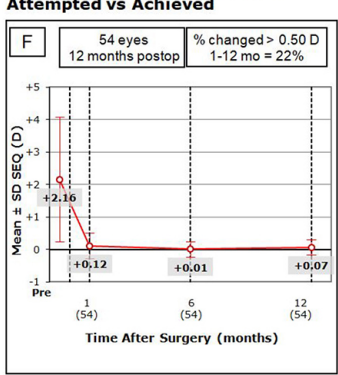

Spherical Equivalent Refraction

Stability

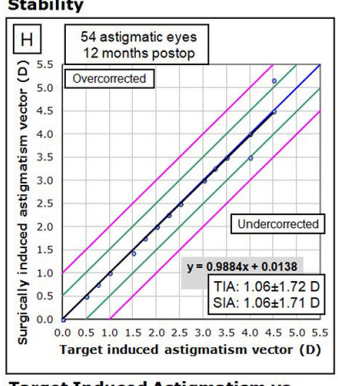

Target Induced Astigmatism vs

Surgically Induced Astigmatism

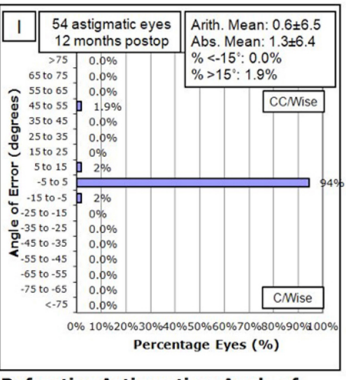

Refractiv

Figure 2: Standard graphs for refractive surgery outcomes. A: Efficacy profile (post-op UDVA vs. pre-op CDVA), B: Difference (loss or gain of lines) between post-op UDVA and pre-op CDVA, C: Safety profile (change in CDVA in form of loss or gain of lines), D: Predictability profile (intended vs. achieved spherical equivalent), E: Accuracy profile (percentage of patients within $\pm 1.00 \mathrm{D}$ ), F: Stability profile (change in mean SEQ over time), G: Refractive astigmatism (percentage of patients within $\pm 1.00 \mathrm{D}$ ), H: TIA vs. SIA, I: Refractive Astigmatism Angle of Error between TIA and SIA. 
Table I: Visual and refractive outcomes in terms of pre- and postoperative UDVA, SEQ and CDVA. Comparison of preoperative values vs. postoperative values at 1, 6, 12 months. The data is presented as means \pm SD and Paired Two-Sample t-Test was applied. P-value of less than $0.05(<0.05)$ is considered as significant.

\begin{tabular}{|c|c|c|c|c|c|c|c|c|}
\hline \multirow{2}{*}{ Parameter } & \multirow{2}{*}{$\begin{array}{c}\text { Preoperative } \\
\text { mean } \\
(M \pm S D)\end{array}$} & \multicolumn{3}{|c|}{$\begin{array}{l}\text { Postoperative mean } \\
\qquad(M \pm S D)\end{array}$} & \multicolumn{4}{|c|}{ p-value (two-tail) } \\
\hline & & 1 Month & 6 Month & 12 Months & $\begin{array}{l}\text { Preoperative } \\
\text { vs. } 1 \text { Mo }\end{array}$ & $\begin{array}{c}\text { Preoperative } \\
\text { vs. } 6 \text { Mo }\end{array}$ & $\begin{array}{l}\text { Preoperative } \\
\text { vs. } 12 \text { Mo }\end{array}$ & $\begin{array}{l}6 \text { Mo vs. } \\
12 \text { Mo }\end{array}$ \\
\hline UDVA (LogMAR) & $0.672 \pm 0.270$ & $0.228+0.180$ & $0.177 \pm 0.175$ & $0.153 \pm 0.182$ & $<0.001$ & $<0.001$ & $<0.001$ & 0.001 \\
\hline SEQ (D) & $2.146 \pm 2.027$ & $0.097 \pm 0.442$ & $0.019 \pm 0.289$ & $0.079 \pm 0.269$ & $<0.001$ & $<0.001$ & $<0.001$ & 0.139 \\
\hline CDVA (LogMAR) & $0.13+0.197$ & $0.171+0.167$ & $0.148+0.180$ & $0.138 \pm 0.183$ & 0.001 & 0.051 & 0.359 & 0.039 \\
\hline
\end{tabular}

Almost $77.8 \%$ (42 out of 54 ) patients had same or better postoperative UDVA when compared to preoperative CDVA. 94.4\% (51 out of 54 ) of patients have UDVA within one line preoperative CDVA.

Safety profile was assessed by observing change (loss or gain of lines) in pre- and postoperative CDVA at 12 months as shown in Figure 2C. Almost $83.3 \%$ (45 out of 54 ) patients had no change in the postoperative CDVA when compared to preoperative CDVA. $7.4 \%$ (4 out of 54) experienced gain of Snellen lines; whereas, 9.3\% (5 out of 54 ) experienced loss of lines. Safety index (ratio of mean postoperative CDVA to mean preoperative (DVA) was calculated to be $0.99 \pm 0.14$.

Scattergram showing the predictability of results was plotted comparing intended vs. achieved spherical equivalent (SEQ) at 12 months. All the achieved points fell within the $\pm 1 D$ intended SEQ range. Figure 2D shows that none of the points fall outside the purple $( \pm 1 D)$ lines. The graph shows that 49 out of 54 patients had postoperative SEQ within $\pm 0.50 \mathrm{D}$ at 12 months.

Accuracy profile is plotted at 12 months and is shown in Figure $2 \mathrm{E}$. Around $91 \%$ (49 out of 54 ) patients had postoperative spherical equivalent within $\pm 0.5 \mathrm{D}$. All the $100 \%$ study patients had postoperative spherical equivalent within \pm $1.00 \mathrm{D}$.

The stability profile was attained by plotting mean SEQ at different time points (preoperative, 1 month, 6 months, 12 months) in Figure 2F. Spherical equivalent showed major improvement from preoperative to postoperative at one month. It remained essentially stable, thereafter, at six months and one year. There were $22.2 \%$ (12 out of 54 ) patients who had change equal to or more than $0.5 \mathrm{D}$ starting from 1 to 12 months.

Astigmatism was compared pre- and postoperatively in Figure 2G. At 12 months duration, $96.3 \%$ (52 out of 54 ) patients had $0.50 \mathrm{D}$ or less astigmatism. Almost $100 \%$ patients had astigmatism within $1.0 \mathrm{D}$ range postoperatively. Scatter plot was plotted comparing target- induced astigmatism (TIA) vs. surgically-induced astigmatism (SIA) at 12 months Figure $2 \mathrm{H}$. Refractive astigmatism angle of error between TIA and SIA is plotted in Figure 2I. All SIA points fell within the $\pm 1 \mathrm{D}$ of TIA. Figure $2 \mathrm{H}$ shows that none of the points fall outside the purple $( \pm 1 \mathrm{D})$ lines. The scatter plot shows that 52 out of 54 patients had postoperative spherical equivalent within $\pm 0.50 \mathrm{D}$ at 12 months. Moreover, $94.4 \%$ (51 out of 54) patients had angle of error with \pm 5 degrees and $98.1 \%$ (53 out of 54 ) of the patients had angle of error within \pm 15 degrees.

\section{DISCUSSION}

Efficacy graph (comparison of preoperative CDVA with postoperative UDVA) shows that $89 \%$ (48 out of 54 ) of the patients had postoperative UDVA, equal to or better than $20 / 40$ at 12 months. Up to $76 \%$ (41 out of 54 ) participants had UDVA equal to or better than 20/32. El-Naggar showed that $85 \%$ patients reached $20 / 20$ at 12 months. ${ }^{17}$ Comparing the efficacy results of both the studies, it is concluded that reaching the target vision of 20/20 in this study was not possible because of inclusion of amblyopic patients (CDVA less than 20/20) in the study. However, the study by ElNaggar had excluded any patients with amblyopia or those with preoperative vision less than 20/30; hence, achieving the target correction of 20/20 post-operatively. Another recent study by $P$ Makinen, that targeted emmetropia, has reported that $91 \%$ of its patients had achieved 20/20 UDVA or better at one month postoperative visit. ${ }^{18}$

Comparing the safety profile; two out of 54 (3.7\%) participants lost two or more lines post-operatively and are under regular follow-up for any need for retreatment. In a study by Kanellopoulos, ${ }^{19} 2.4 \%$ lost two or more lines and $5.3 \%$ lost one or more lines at 24 months. Similar study by Llovet et al. showed $5.7 \%$ patients losing 2 or more lines at the end of one year. ${ }^{20}$ Another study that was conducted in Turkey by Demir et al. reported that $12.6 \%$ patients lost one line in CDVA at four-year follow-up. ${ }^{21}$

This study showed excellent predictability as $100 \%$ of the patients had achieved results within $\pm 1.0 \mathrm{D}$ of the attempted refraction. This aspect was comparable to study by El-Naggar, which also documented $100 \%$ patients within $\pm 1.0 \mathrm{D}$ of attempted refractive correction. ${ }^{17}$ However, 95\% of their participants had reached within $\pm 0.50 \mathrm{D}$ of attempted refraction at 12 months as compared to $91 \%$ of these patients at 12 months. Similarly, in the study by Demir, $89 \%$ patients were within $\pm 1 \mathrm{D}$ of emmetropia at end of 1 year. ${ }^{21}$ 
The accuracy in this study was comparable to other international studies like the study by Kenellopoulos, which was conducted on similar laser systems. At 24 months, 75.5\% patients were within $\pm 0.50 \mathrm{D}$ range and $94.4 \%$ were in $\pm 1.00 \mathrm{D}$ range. ${ }^{20}$ Motwani and Pei reported $77.9 \%$ patients within $\pm 0.50 \mathrm{D}$ range at six months. ${ }^{22}$

This study showed that $100 \%$ patients were within $\pm 1.00 \mathrm{D}$ range where as $91 \%$ were within $\pm 0.50 \mathrm{D}$ range at the end of 12 months. These results are also comparable to the recent study by Makinen. All of their patients were within $\pm 1.0 \mathrm{D}$ at end of 1 month and $88 \%$ were within $\pm 0.5 \mathrm{D}$ range. ${ }^{18}$

El-Naggar revealed that $85 \%$ of their patients had less than or equal to $1.0 \mathrm{D}$ astigmatism postoperatively $i^{17}$ whereas, $100 \%$ of patients in this study fell in this category.

This study was restrained by a shorter duration of follow-up. Longer duration study will be needed to analyse long term regression trends as typically associated with hyperopia. Studying higher order aberrations and effect of treatment on quality of life will give further understanding of the overall effect of treatment on patients' day-to-day life.

\section{CONCLUSION}

LASIK, performed to correct hyperopia, demonstrated satisfactory outcomes in terms of safety, efficacy, stability, accuracy and predictability. LASIK is an effective treatment option for correcting moderate hyperopia.

\section{ETHICAL APPROVAL:}

This study was carried out after obtaining approval from the Institutional Review Board and Ethical Committee of Armed Forces Institute of Ophthalmology.

\section{PATIENTS' CONSENT:}

Informed consent were taken from all patients participated in the study.

\section{CONFLICT OF INTEREST:}

The authors declared no conflict of interest.

\section{AUTHORS' CONTRIBUTION:}

$\mathrm{SH}$ : Conception and design of the work.

MI: Final approval of manuscript.

AF: Drafting of the work.

SFM: Interpretation of data.

QH: Data analysis.

SA: Data acquisition.

\section{REFERENCES}

1. Jackson WB, Tuan KM, Mintsioulis G. Aspheric wavefront-guided LASIK to treat hyperopic presbyopia: 12-month results with the VISX platform. J Refract Surg 2011; 27(7):519-29. doi: 10.3928/1081597X-20101110-02.

2. Gil-Cazorla R, Teus MA, de Benito-Llopis L, Mikropoulos DG.
Femtosecond laser vs mechanical microkeratome for hyperopic laser in situ keratomileusis. Am J Ophthalmol 2011; 152(1):16-21. doi: 10.1016/j.ajo.2011.01.009.

3. Gómez de Liaño-Sánchez R, Borrego-Hernando R, Franco-Iglesias G, Gómez de Liaño-Sánchez P, Arias-Puente A.

Strabismus and diplopia after refractive surgery. Arch Soc Esp Oftalmol 2012 Nov; 87(11):363-7. doi: 10.1016/j.oftal.2011.12.006

4. Frings A, Intert E, Steinberg J, Druchkiv V, Linke SJ, Katz T. Hyperopia shows the strongest association with LASIK retreatment. Acta Ophthalmol 2018; 96(3):e404. doi: 10.1111/aos.13486.

5. Fatseas G, Stapleton F, Versace P. Role of percent peripheral tissue ablated on refractive outcomes following hyperopic LASIK. PLoS One 2017; 12(2):e0170559. doi: 10.1371/journal.pone.0170559.

6. Aslanides IM, Mukherjee AN. Adjuvant corneal crosslinking to prevent hyperopic LASIK regression. Clin Ophthalmol 2013; 7:637-41. doi: 10.2147/OPTH.S39796.

7. Eid AM, Abdelghany AA, Abdelhalim AS, Sabry AM. Concurrent laser-assisted in situ keratomileusis with high-fluence cross-linking versus laser-assisted in situ keratomileusis only in treatment of hyperopia. J Egypt Ophthalmol Soc 2019; 112(4):117-21. doi: 10.4103/ejos.ejos_38_19

8. Basmak H, Sahin A, Yildirim N, Papakostas TD, Kanellopoulos AJ. Measurement of angle kappa with synoptophore and orbscan II in a normal population. J Refract Surg 2007; 23(5):456-60.

9. Rios LC, Silva PGD, Canamary Junior AM, Rodrigues $P$, Regatieri CVS, Campos $\mathrm{M}$, et al. Centration in refractive surgery. Arq Bras Oftalmol 2020; 83(1):76-81. doi: 10.5935/ 0004-2749.20200014.

10. Sales CS, Manche EE. One-year eye-to-eye comparison of wavefront-guided versus wavefront-optimized laser in situ keratomileusis in hyperopes. Clin Ophthalmol 2014; 8:2229-38. doi: 10.2147/OPTH.S70145.

11. He L, Liu A, Manche EE. Wavefront-guided versus wavefront-optimized laser in situ keratomileusis for patients with myopia: a prospective randomized contralateral eye study. Am J Ophthalmol 2014; 157(6):1170-8. doi: 10.1016/j.ajo.2014.02.037.

12. Frings A, Steinberg J, Druchkiv V, Linke SJ, Katz T. Role of preoperative cycloplegic refraction in LASIK treatment of hyperopia. Graefes Arch Clin Exp Ophthalmol 2016; 254(7):1399-404. doi: 10.1007/s00417-016-3308-z.

13. Kanellopoulos AJ. Managing highly distorted corneas with topography-guided treatment. ISRS/AAO 2007 subspecialty day/refractive surgery syllabus. Section II: Ablation Strategies. 2007 Nov 10:13-5.

14. Wachler BSB, Korn TS, Chandra NS, Michel FK. Decentration of the optical zone: centering on the pupil versus the coaxially sighted corneal light reflex in LASIK for hyperopia. J Refract Surg 2003; 19(4):464-5.

15. Waring GO, Reinstein DZ, Dupps WJ Jr, Kohnen T, Mamalis $\mathrm{N}$, Rosen ES, et al. Standardised graphs and terms for refractive surgery results. J Refract Surg 2011; 27(1):7-9. doi: 10.3928/1081597X-20101116-01.

16. Stulting RD, Dupps WJ Jr, Kohnen T, Mamalis N, Rosen ES, 
Koch DD, et al. Standardised graphs and terms for refractive surgery results. Cornea 2011; 30(8):945-7. doi: 10.1097/ICO.0b013e31820a0e53.

17. El-Naggar MT, Hovaghimian DG. Assessment of refractive outcome of femtosecond-assisted LASIK for hyperopia correction. Electron Physician 2017; 9(3):3958-65. doi: $10.19082 / 3958$.

18. Makinen P, Huhtala A, Pietila J, Nattinen J, Rajala T, Salmenhaara $\mathrm{K}$, et al. Patient satisfaction and self-reported dry eye symptoms in hyperopic patients treated with femtosecond laser in situ keratomileusis. Clin Ophthalmol 2019; 13:741-54. doi: 10.2147/OPTH.S195991.

19. Kanellopoulos AJ. Topography-guided hyperopic and hyperopic astigmatism femtosecond laser-assisted LASIK: longterm experience with the $400 \mathrm{~Hz}$ eye-Q excimer platform.
Clin Ophthalmol 2012; 6:895-901. doi: 10.2147/OPTH. S23573.

20. Llovet F, Galal A, Benitez-del-Castillo JM, Ortega J, Martin C, Baviera J. One-year results of excimer laser in situ keratomileusis for hyperopia. J Cataract Refract Surg 2009; 35(7):1156-65. doi: 10.1016/j.jcrs.2009.03.014.

21. Demir G, Sucu ME, Yıldırım Y, Tülü B, Özveren M, Kepez Yıldız B. Long-term assessment of visual and refractive outcomes of laser in situ keratomileusis for hyperopia using the AMARIS ${ }^{\circledR} 750$ S excimer laser. J Fr Ophtalmol 2019; 42(7):703-10. doi: 10.1016/j.jfo.2019.02.006.

22. Motwani M, Pei R. Treatment of moderate-to-high hyperopia with the wave light allegretto 400 and EX500 excimer laser systems. Clin Ophthalmol 2017; 11:999-1007. doi: 10.2147/OPTH.S136061. 